PREPARED FOR THE U.S. DEPARTMENT OF ENERGY, UNDER CONTRACT DE-AC02-76CH03073

PPPL-3654

PPPL-3654

UC-70

Magnetic Field Generation through Angular Momentum Exchange between Circularly Polarized Radiation and Charged Particles

by

G. Shvets, N.J. Fisch, and J.-M. Rax

January 2002

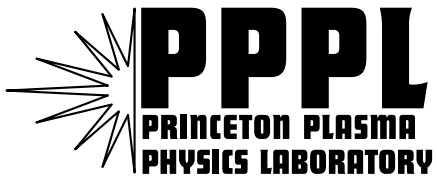

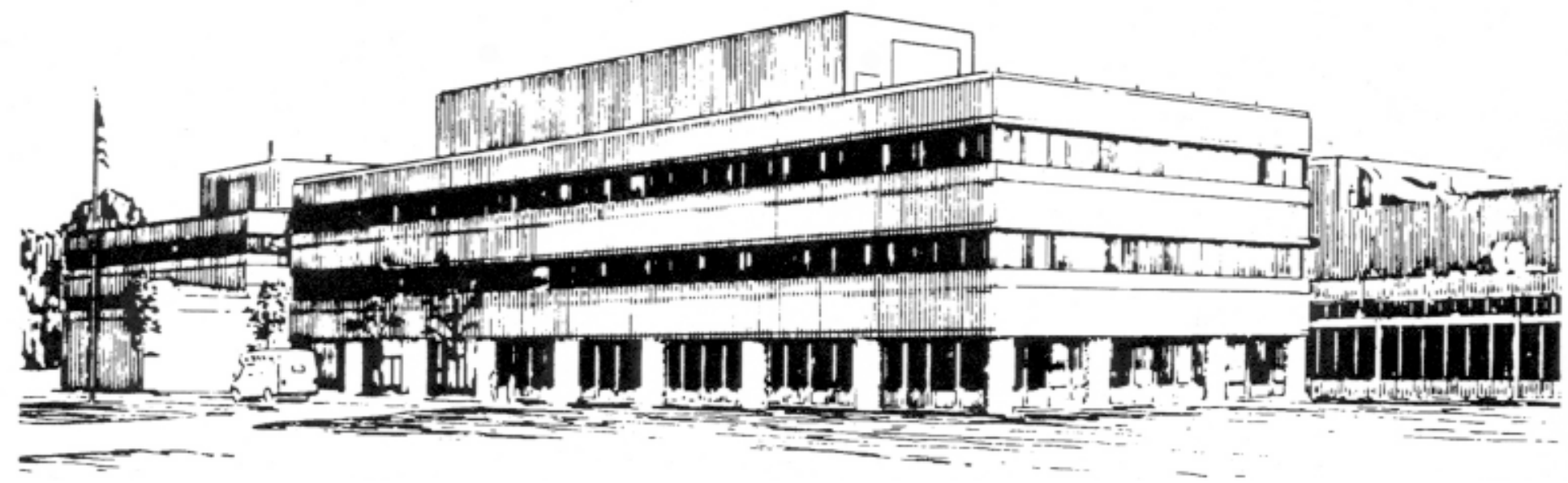

PRINCETON PLASMA PHYSICS LABORATORY PRINCETON UNIVERSITY, PRINCETON, NEW JERSEY 


\section{PPPL Reports Disclaimer}

This report was prepared as an account of work sponsored by an agency of the United States Government. Neither the United States Government nor any agency thereof, nor any of their employees, makes any warranty, express or implied, or assumes any legal liability or responsibility for the accuracy, completeness, or usefulness of any information, apparatus, product, or process disclosed, or represents that its use would not infringe privately owned rights. Reference herein to any specific commercial product, process, or service by trade name, trademark, manufacturer, or otherwise, does not necessarily constitute or imply its endorsement, recommendation, or favoring by the United States Government or any agency thereof. The views and opinions of authors expressed herein do not necessarily state or reflect those of the United States Government or any agency thereof.

\section{Availability}

This report is posted on the U.S. Department of Energy's Princeton Plasma Physics Laboratory Publications and Reports web site in Fiscal Year 2002. The home page for PPPL Reports and Publications is: http://www.pppl.gov/pub_report/

DOE and DOE Contractors can obtain copies of this report from:

U.S. Department of Energy

Office of Scientific and Technical Information

DOE Technical Information Services (DTIS)

P.O. Box 62

Oak Ridge, TN 37831

Telephone: (865) 576-8401

Fax: (865) 576-5728

Email: reports@adonis.osti.gov

This report is available to the general public from:

National Technical Information Service

U.S. Department of Commerce

5285 Port Royal Road

Springfield, VA 22161

Telephone: 1-800-553-6847 or

(703) 605-6000

Fax: (703) 321-8547

Internet: http://www.ntis.gov/ordering.htm 


\title{
Magnetic Field Generation through Angular Momentum Exchange Between Circularly Polarized Radiation and Charged Particles
}

\author{
G. Shvets and N. J. Fisch \\ Princeton Plasma Physics Laboratory, Princeton University, Princeton, NJ 08543 \\ J.-M. Rax \\ LPGP, bat 212, Universite-Paris XI, Orsay Cedex 91405, France
}

\begin{abstract}
The interaction between circularly polarized $(\mathrm{CP})$ radiation and charged particles can lead to generation of magnetic field through an inverse Faraday effect. The spin of the circularly polarized electromagnetic wave can be converted into the angular momentum of the charged particles so long as there is dissipation. We demonstrate this by considering two mechanisms of angular momentum absorption relevant for laser-plasma interactions: electron-ion collisions and ionization. The precise dissipative mechanism, however, plays a role in determining the efficiency of the magnetic field generation.
\end{abstract}

The interaction between circularly polarized $(\mathrm{CP})$ radiation and charged particles occurs in nature and in the laboratory. For example, radio pulsars are believed to be rotating magnetized neutron stars $[1,2]$ which produce powerful circularly polarized electromagnetic waves along the rotation axis. Recent laboratory experiments [3-5] demonstrated that large quasi-static magnetic fields can be produced when a circularly polarized laser pulse interacts with the plasma. This method of magnetic field generation can be utilized for a hybrid inertial-magnetic fusion confinement [6].

The circularly polarized EM radiation has non-vanishing angular momentum associated 
with the photon spin. In the course of the wave-particle interaction, this spin can be transferred to the medium [7]. When a medium contains free electrons, azimuthal electric current can be induced, and a magnetic field is generated through the inverse Faraday effect (IFE). IFE in an arbitrary gyrotropic medium was first predicted on the basis of a thermodynamic argument by Pitaevskii [8], and later re-discovered in plasmas by Deschamps et. al. and Steiger and Woods [9]. They argued that the $\mathrm{CP}$ wave with a normalized vector potential $a_{0}=e A / m c^{2}$ and frequency $\omega$ induces an axial magnetic field $B_{0}=\frac{m c}{2 e} \frac{a^{2} \omega_{p}^{2}}{\omega}$, where $\omega_{p}=\left(4 \pi e^{2} n / m\right)^{1 / 2}$ is the electron plasma frequency. The conditions for producing this azimuthal flow have not been addressed in Ref. [9] and in subsequent publications on the subject $[4,10-12]$. For example, the classic IFE $\left(B_{z} \propto a_{0}^{2}\right)$ cannot occur in a pre-formed collisionless plasma.

The purpose of this Letter is to demonstrate that strong axial magnetic fields proportional to the laser intensity can be generated in the plasma by an intense CP laser pulse owing to dissipative effects which result in the absorption of the laser angular momentum. We present examples of two such effects: electron collisions and ionization inside the laser pulse. It is of importance also precisely how these dissipative effects enter. Note that a higher-order IFE $\left(B_{z} \propto a_{0}^{4}\right)$ is possible even in the absence of the angular momentum absorption due to relativistic effects [13].

First, note that a classical CP electromagnetic wave indeed possesses angular momentum given by its spin. The vector potential of the right-hand polarized EM field is given by the following expression:

$$
\frac{e \vec{A}}{m c^{2}}=\left[\frac{\vec{e}_{+}}{2} a_{0}(r)+i \frac{\vec{e}_{z}}{2 k} \frac{\partial a_{0}}{\partial r}\right] e^{i \theta}+c . c .
$$

where $\theta=k z-\omega t+\phi$ is the wave phase, $\phi=\tan ^{-1} y / x$ is the angle of the position phasor, and $\vec{e}_{+}=\vec{e}_{r}+i \vec{e}_{\phi}$. The normalized electric $\vec{e}=(-1 / c) \partial \vec{a} / \partial t$ and magnetic $\vec{b}=\nabla \times \vec{a}$ fields define the normalized Poynting flux $\vec{p}=\vec{e} \times \vec{b}$ :

$$
\vec{p}=k^{2}\left[a_{0}^{2} \vec{e}_{z}-\frac{1}{2 k} \frac{\partial a_{0}^{2}}{\partial r} \vec{e}_{\phi}\right],
$$


where we have assumed a tenuous plasma $\omega_{p}^{2} \ll \omega^{2}$ and a broad laser pulse with the focal spot $\sigma \gg k^{-1}$. The transverse profile of the pulse is assumed Gaussian which is a valid assumption in the vicinity of the laser focus. The first term is the Poynting flux in the forward direction and the second term is associated with the photon spin. Therefore, the Poynting flux of the laser pulse spirals around the $z$ - axis. This spiraling motion is precisely the classical equivalent of the photon spin in quantum mechanics. The azimuthal timeaveraged component of the Poynting flux vanishes for linear polarization, and reverses its sign for the left-polarized EM wave (polarity is reversed by replacing $\vec{e}_{+}$by $\vec{e}_{-}=\vec{e}_{r}-i \vec{e}_{\phi}$ ).

To calculate magnetic field generation in a weakly collisional plasma, we integrate the electron motion in the field of a transversely non-uniform laser pulse. To examine the electron motion in the EM wave, it is convenient to use the Lagrangian description [14]: let $\vec{\xi}\left(t, \vec{x}_{0}\right)$ be the displacement of an electron initially located at $\vec{x}=\vec{x}_{0}$, which, for $a_{0}<1$, satisfies the non-relativistic equation of motion

$$
\frac{\partial \dot{\vec{\xi}}}{\partial t}+\gamma \dot{\vec{\xi}}=-\frac{e}{m}\left(\vec{E}+\frac{\dot{\vec{\xi}} \times \vec{B}}{c}\right)
$$

where $\vec{E}$ and $\vec{B}$ are evaluated at $\vec{x}_{0}+\vec{\xi}$. Collisions are implemented in our model through the friction coefficient $\gamma$.

It is convenient to separate the electron displacement into the high- and low-frequency components $\vec{\xi}_{1}\left(t, \vec{x}_{0}\right)$ and $\vec{\xi}_{2}\left(t, \vec{x}_{0}\right)$, respectively. Under a weakly relativistic assumption $a_{0}^{2} \ll 1, \vec{\xi}_{1}\left(t, \vec{x}_{0}\right)$ is of order $a_{0}: \dot{\vec{\xi}}_{1} / c \approx \vec{a}+\gamma / \omega^{2} \partial \vec{a} / \partial t$. Note that $\dot{\vec{\xi}}_{1} \cdot \vec{E} \neq 0$, resulting in the collisional damping of the EM wave (inverse bremsstrahlung). The inverse bremsstrahlung (IB) rate $\gamma_{w}$ is given by $\gamma_{w}=\gamma_{f} \omega_{p}^{2} / \omega^{2}[15]$, where $\gamma_{f}$ is the fast collisions frequency.

Determining the appropriate collision frequency $\gamma$ or $\gamma_{f}$ is not straightforward. For example, for electron-ion collisions, in the limit of weak EM wave $a_{0} c \ll v_{t h}$, the averaged over the Maxwellian distribution high-frequency collision rate is given by $\gamma_{f}=\left(4 \pi Z_{i} e^{4} n_{0} \Lambda_{f} / m^{1 / 2} T_{e}^{3 / 2}\right)$, where $T_{e}$ is the electron temperature, $Z_{i}$ is the ion charge (taken to be unity for singly ionized ions), and $\Lambda_{f}=\ln \left(2^{5 / 2} T_{e}^{3 / 2} / \Gamma^{5 / 2} \omega_{0} Z_{i} e^{2} m^{1 / 2}\right)$, where $\Gamma=0.577 \ldots$ is the Euler constant [17]. For $\omega \gg \omega_{p}, \Lambda_{f}$ is smaller than the standard 
Coulomb logarithm because only collisions with impact parameters $\rho<v / \omega$ contribute to the inverse bremsstrahlung. Even greater reduction of the IB occurs for strong electromagnetic waves with $a_{0} c \geq v_{t h}: \gamma_{f} \propto\left(a_{0} c / v_{t h}\right)^{-3}[16]$.

A second characteristic collision frequency $\gamma_{s}$ describes the scattering rate of the slow oscillation center drifts. For electron-ion collisions in a hot plasma with $v_{t h}>a_{0} c$, it is given by $\gamma_{s}=4 \pi Z_{i} e^{4} n_{0} /\left(m^{1 / 2} T_{e}^{3 / 2}\right) \Lambda$. Interestingly, $\gamma_{s}$ can be substantially higher than $\gamma_{f}$ because both large and small angle collisions contribute to $\gamma_{s}$, but only the large-angle collisions contribute to $\gamma_{f}[17]$. The difference between the two collisional rates is small when the laser intensity is weak: only the Coulomb logarithm is different for $\gamma_{s}$ and $\gamma_{f}$. However, in a strongly-illuminated plasma, $\gamma_{s} / \gamma_{f} \sim\left(v_{\text {osc }} / v_{\text {th }}\right)^{2} \gg 1[18]$.

To calculate the time-averaged electron current $\vec{J}_{e}$, note that in the Lagrangian formulation the total time-averaged electron velocity

$$
v_{s \phi}(\vec{x})=\int d^{3} x_{0}\left\langle\dot{\xi}_{\phi} \delta^{3}\left[\vec{x}-\vec{x}_{0}-\vec{\xi}\right]\right\rangle
$$

consists of two components $\vec{v}_{s}=\vec{v}_{s}^{c}+\vec{v}_{s}^{m}$ : the slow convective drift of the oscillation centers $\vec{v}_{s}^{c}=\dot{\vec{\xi}}_{2}$ and the magnetization drift $\vec{v}_{s}{ }^{m}=-\left(\vec{\xi}_{1} \cdot \nabla\right) \dot{\vec{\xi}}_{1}$. (We note that if $\nabla \cdot \vec{A}=0$ is not satisfied as in Refs. [11,12], then an additional term en $\dot{\xi}_{1 \phi}\left(\nabla \cdot \vec{\xi}_{1}\right)$ appears to contribute to magnetic field generation. This leads to an incorrect conclusion that magnetic field can be generated in a homogeneous plasma in the absence of dissipative processes).

Substituting $\vec{\xi}_{1}$ into From Eq. (3), the time-averaged equation for $\vec{\xi}_{2}$ is derived:

$$
\begin{aligned}
& \frac{\partial \dot{\vec{\xi}}_{2}}{\partial t}+\gamma_{s} \dot{\vec{\xi}}_{2}=-c^{2} \vec{\nabla}\left(\frac{a_{0}^{2}}{2}\right)-\frac{e \vec{E}_{s}}{m}+ \\
& \frac{\partial}{\partial t}\left[\left(\vec{\xi}_{1} \cdot \nabla\right) \dot{\vec{\xi}}_{1}\right]+\gamma_{f}\left[\left(\vec{\xi}_{1} \cdot \nabla\right) \dot{\vec{\xi}}_{1}-\dot{\vec{\xi}}_{1} \times \nabla \times \vec{\xi}_{1}\right] .
\end{aligned}
$$

Note that we have substituted the appropriate collision frequency $\gamma_{s}$ into the LHS of Eq. (4). The $-\nabla\left(a_{0}^{2} / 2\right)$ term in the RHS of Eq. (4) is the standard ponderomotive force which does not project onto $\phi$-direction for azimuthally symmetric laser pulse. Electric field $\vec{E}_{s}$ is induced by the quasi-static magnetic field through Faraday's law; it vanishes in steady state, and is in general small when the spotsize $\sigma<c / \omega_{p}$. The last term in the RHS of Eq. (4) also 
does not project onto $\phi$-direction. The remaining term, $\partial_{t}\left[\left(\vec{\xi}_{1} \cdot \nabla\right) \dot{\vec{\xi}}_{1}\right]$, determines, in the absence of collisions, the oscillation center drift: $\dot{\vec{\xi}}_{2}=\left(\vec{\xi}_{1} \cdot \nabla\right) \overrightarrow{\vec{\xi}}_{1}$. This drift exactly cancels the magnetization drift: $\vec{v}_{s}=\dot{\vec{\xi}}_{2}-\left(\vec{\xi}_{1} \cdot \nabla\right) \dot{\vec{\xi}}_{1}=0$. Therefore, without collisions there is no time-averaged azimuthal current and, consequently, no axial magnetic field. With collisions, in steady state, $\dot{\vec{\xi}}_{2}=0$, and the time-averaged electron fluid velocity $\vec{v}_{s}=-\left(\vec{\xi}_{1} \cdot \nabla\right) \dot{\vec{\xi}}_{1}$ drives magnetic field.

Note that the convective velocity $\vec{v}_{s}^{c}=\dot{\vec{\xi}}_{2}=\left(\vec{\xi}_{1} \cdot \nabla\right) \dot{\vec{\xi}}_{1}$ represents a real physical drift of the oscillation center of an electron subjected to the transversely inhomogeneous CP electromagnetic wave which has been, to our knowledge, overlooked. To visualize this drift, we performed a numerical simulation of the single-particle motion in the prescribed electromagnetic field of a laser pulse which is adiabatically turned on over several laser periods (see Fig. 1 for parameters). Azimuthal displacement $y=r \phi$ of the electron through approximately 15 laser periods is plotted in Fig. 1 without (solid line) and with (dashed line) collisions. The thin solid line drawn through the displacement maxima in the collisionless case $(\gamma=0)$ is a visual aid indicating the drift of the oscillation centers in the negative $\phi$ direction. Its slope is determined by the oscillation center (OC) drift velocity $\dot{\xi}_{2 y}=\left(c^{2} / 2 \omega\right) \partial_{r} a_{0}^{2}$ derived above. Since the current produced by the OC drift cancells the magnetization drift $\vec{v}_{s y}^{m}=-\left(\vec{\xi}_{1} \cdot \nabla\right) \dot{\vec{\xi}}_{1 y}$, no azimutal current is produced without collisions. With collisions, for $\gamma / \omega=0.1$, the OC drift is dissipated by the frictional force. For simplicity, the subtlety of $\gamma_{s} \neq \gamma_{f}$ is neglected in the simulation.

The equation for the total azimuthal component of the fluid velocity is given by $\partial_{t} v_{s \phi}+\gamma_{s} v_{s \phi}=-\gamma_{s}\left\langle\left(\vec{\xi}_{1} \cdot \nabla\right) \dot{\xi}_{1 \phi}\right\rangle-e E_{s \phi} / m$. This can now be used together with Ampere's law to obtain an equation for the axial component of the magnetic field:

$$
\frac{\partial}{\partial t}\left(\nabla_{r}^{2} B_{z}-k_{p}^{2} B_{z}\right)+\gamma_{s} \nabla_{r}^{2} B_{z}=\gamma_{s} \nabla_{r}^{2} B_{0}
$$

where $k_{p}=\omega_{p} / c, \nabla_{r}^{2}=r^{-1} \partial_{r}\left(r \partial_{r}\right)$ and $B_{0}=-2 \pi e n c a_{0}^{2} / \omega$ is the steady-state magnetic field in the plasma, as calculated by Steiger and Woods [9], and we assumed that the laser pulse which is transversely thin and varies slowly in $z$ and $t$ (typical for tightly-focused laser 
pulses). For large times magnetic field $B_{z}$ approaches $B_{0}(r)$. Note that magnetic field can never exceeds $B_{0}$, no matter how fast is the rate of the angular momentum loss by the EM wave. This is simply because, while the electron plasma experiences larger torque for larger $\gamma_{f}$, it also experiences a larger friction force.

Note that the appearance in the plasma of net angular momentum is due to the unequal effect of collisions on the cancelling drifts, namely the oscillation center drift and the magnetization drift. We can compare the appearance of angular momentum due to a circularly polarized wave with the appearance of linear momentum, or current, due to an electrostatic wave with large phase velocity $v_{\mathrm{ph}} \gg v_{\mathrm{th}}[19]$. In both cases, the absence of collisions results in cancelling currents. The electrostatic wave carries no momentum in the sense that were it to damp by means of quasilinear theory within a plasma, say on electrons, as a result of this damping, the electron distribution function would conserve momentum but not energy. Therefore, while the wave may appear to give momentum to resonant electrons on the tail of the electron velocity distribution, there is in fact an entirely cancelling current by the nonresonant slow electrons. However, should the nonresonant bulk electrons collide more frequently than the fast tail electrons, the tail electrons will carry a current. Collisions are similarly necessary for an electromagnetic wave to drive current [20], although in that case there is no bulk current to cancel, but rather an asymmetry is induced in the collisions of the counter-propagating electron tails. Similarly, other current drive mechanisms also require non-vanishing collisions [21].

Another important thing to note is that the turn-on time of the magnetic field $B_{z}$ is determined solely by $\gamma_{s}$ (i. e. by the small-angle collisions). The power dissipation rate due to collisions is, however, determined by $\gamma_{f}$ (i. e. by the large-angle collisions). One can pose the following question: how much dissipated power per unit volume $d P / d V$ is required to maintain a given magnetic field $B$. The answer is given by an efficiency formula

$$
\frac{B}{d P / d V}=\frac{e}{2 m c \omega \gamma_{f}}
$$

which implies that in a strongly illuminated plasmas one can achieve a high efficiency of 
B-field generation due to the reduction in the IB rate. The turn-on time would only increase slightly since $\gamma_{s} \gg \gamma_{f}$. Note that, as with the current drive efficiencies [21], this efficiency scales inversely with collision rate. The current drive analogy is appropriate when inverse bremsstrahlung is the principal absorption mechanism.

Collisions is not the only mechanism which enables angular momentum absorption and magnetic field generation. Gas ionization in the presence of EM wave also enables IFE. Moreover, we find that it is not necessary for the ionization to be done by the CP pulse itself: electrons can be released from the atoms through any ionization process as long as it occurs inside the pulse. This process is purely classical, and should be distinguished from the de magnetization of gases and solids achieved by the direct optical pumping $[22,23]$.

Assume that every electron which is released from an atom has a vanishing velocity at the time of its release. Lagrangian description of the continuously ionized plasma is still possible, only now the displacement of the $j$ 's electron $\vec{\xi}^{(j)}(t) \equiv \vec{\xi}\left(t, t^{\prime}, \vec{x}_{0}\right)$ becomes a function of its release time $t^{\prime}$. For simplicity, assume a stratified geometry, where $x$ corresponds to the radial position $r$ and $y$ corresponds to the azimuthal distance $r \phi$. Neglecting the inductive electric field (for $\sigma<c / \omega_{p}$ ), by conservation of canonical momentum, the azimuthal velocity of the $j$ 's electron at the location $\vec{x}=\vec{x}_{0}+\vec{\xi}^{(j)}$ at time $t$ is

$$
\beta_{y}^{(j)}=a_{y}(t, \vec{x})-a_{y}\left(t^{\prime}, \vec{x}_{0}\right)
$$

In a preformed plasma, only the first term in the RHS of Eq. (7) remains. The second term in the RHS of Eq. (7) arises because the $j$ 's electron is born in a non-vanishing vector potential $a_{y}\left(t^{\prime}, \vec{x}_{0}\right)$. It is absent for electrons produced before the arrival of the laser pulse. In a completely preformed plasma, in the absence of collisions, there is no azimuthal current even inside the laser pulse. However, finite azimuthal current is left in the wake of an ionizing laser pulse, creating a long solenoid of magnetic field behind the pulse.

Total azimuthal current density $n \beta_{y}$ at the space-time point $(t, \vec{x})$ can be expanded to the lowest order in $\xi_{x}$ as

$$
n \beta_{y} \approx-\int_{-\infty}^{t} d t^{\prime}\left(\left[\dot{p} n_{G}\right] a_{y}\left(t^{\prime}, \vec{x}\right)+\vec{\xi} \cdot \nabla\left[a_{y}\left(t^{\prime}, \vec{x}\right) \dot{p} n_{G}\right]\right)
$$


where $\dot{p} n\left(\vec{x}_{0}\right)$ is the probability of creating an electron in the vicinity of $\vec{x}_{0}$ per unit volume per unit time. The density of neutral gas is $n_{G}$, and the ionization occurs with the probability $\dot{p}$ as the result of, e. g., tunneling or multi-photon ionization. Whichever mechanism prevails depends on the laser parameters. The assumption of the electron release at rest is reasonable for most ionization scenarios.

The first term in the RHS of Eq. (8), although linear in the laser field, is negligibly small if the ionization takes place over several laser periods, as we are going to assume. The vanishing of the first term in the RHS of Eq. (8) is due to the phase-mixing of the velocities of electrons released during different phases of the laser vector potential. The magnitude of the linear term is of the order $\exp \left[-\omega_{0}^{2} \tau_{i}^{2}\right]$ assuming that $\dot{p} \propto \exp \left[-t^{2} / \tau_{i}^{2}\right]$. It is the second, nonlinear term which can produce a sizable contribution to the azimuthal current due to the fact that the time-averaged product $\left\langle\xi_{x} a_{y}\right\rangle$ does not vanish. Physically, electron displacement $\vec{\xi}$ due to the laser field ensures that two different electrons $j$ and $k$, released at different times $t_{j}$ and $t_{k}+\pi / \omega$ at the same location $\vec{x}_{0}$, end up spatially separated after the passage of the laser pulse. Had they not been spatially separated, their respective contributions to the plasma angular momentum, $\dot{\xi}_{y}^{(j)} \times x^{(j)}$ and $\dot{\xi}_{y}^{(k)} \times x^{(k)}$, would have cancelled.

Since we are concerned with obtaining the angular velocity of order $c|a|^{2}(k \sigma)^{-1}$, and there is already one $\partial / \partial x$ derivative in the nonlinear term of Eq. (8), it can be assumed that

$$
\frac{\vec{\xi}^{(j)}}{c}=\int_{t^{\prime}}^{t} d t^{\prime \prime}\left[\vec{a}\left(t^{\prime \prime}\right)-\vec{a}\left(t^{\prime}\right)\right],
$$

where the dependence of $\vec{a}$ on $\vec{x}$ is implied. Assuming that $a_{x}=a_{0} \cos (\omega t-k z), a_{y}=$ $a_{0} \sin (\omega t-k z)$, and $a_{z}=\partial_{x} a_{0} \sin (\omega t-k z) / k$, it can be shown that

$$
n \beta_{y}(t)=-\frac{c}{2 \omega} \frac{\partial}{\partial x} \int_{-\infty}^{t} d t^{\prime}\left[\dot{p} n_{G}\right] a^{2} .
$$

Integrating over the transverse dimensions yields the volume density of the angular momentum left behind the laser pulse:

$$
\mathcal{L}_{z}(\vec{x})=\frac{m c^{2}}{\omega} \int_{-\infty}^{+\infty} d t^{\prime}\left(\frac{d n}{d t^{\prime}}\right) a^{2}\left(t^{\prime}, \vec{x}\right) .
$$


A straightforward calculation confirms that Eq. (11) is identically equal to loss rate of the laser angular momentum. From Ampere's law and Eq. (10), the magnetic field left behind the laser pulse is given by

$$
\frac{e B_{z}}{m c}=\frac{-1}{2 \omega} \int_{-\infty}^{t} d t^{\prime}\left(\frac{d \omega_{p}^{2}}{d t^{\prime}}\right) a^{2}\left(t^{\prime}, \vec{x}\right) .
$$

According to Eqs. $(11,12)$, the magnetic field is simply proportional to the angular momentum lost by the laser pulse. Note that, although electrostatic wakes behind the ionizing laser pulse have been studied [24], Eq. (12) describes a magnetic wake The present calculation demonstrates that, during ionization, magnetic fields can be produced on a sub-picosecond time scale in a collisionless plasma.

Consider the following example: a $50 \mu \mathrm{J} / 30 \mathrm{fs}$ laser pulse with the $\lambda=0.8 \mu \mathrm{m}$ wavelength is focused to the $d=6 \mu \mathrm{m}$ diameter onto the helium gas at atmospheric pressure. The peak intensity $I=4 \times 10^{15} \mathrm{~W} / \mathrm{cm}^{2}$ corresponds to $a^{2} \approx 10^{-3}$, and the plasma density (assuming single-stage ionization) $n=5.4 \cdot 10^{19} \mathrm{~cm}^{-3}$. From Eq. (12), the peak magnetic field $B_{0} \approx 2.5 \mathrm{kG}$.

In summary, the magnetic field generation through the inverse Faraday effect requires a dissipative mechanism of coupling the angular (spin) momentum of the laser to the plasma, but the precise nature of the dissipation can enter importantly. Two examples, collisional absorption and ionization, are considered. Ionization-induced IFE opens the possibility of generating large magnetic fields on an ultra-short time scale. 


\section{REFERENCES}

[1] F. C. Michel and H. Li, Phys. Reports 318, 227 (1999).

[2] A. Melatos and D. B. Melrose, Mon. Not. R. Astron. Soc. 279, 1168 (1996).

[3] Y. Horovitz et. al., Phys. Rev. Lett. 78, 1707 (1997).

[4] T. Lehner, Europhys. Lett. 50, 480 (2000).

[5] M. Tatarakis et. al., "Measurements of the Inverse Faraday effect in high intensity laser produced plasmas", submitted to Phys. Rev. Lett. (2000).

[6] E. Kolka, S. Eliezer, and Y. Pais, Phys. Let. A 180, 132 (1993).

[7] A. I. Sadovskii, Uch. Zap. Imp. Yur'evsk Un-ta., No. 1, 1-126 (1899).

[8] L. P. Pitaevskii, Sov. Phys. JETP 12, 1008 (1961).

[9] J. Deschamps et. al., Phys. Rev. Lett. 25, 1330 (1970); A. D. Steiger and C. H. Woods, Phys. Rev. A 5, 1467 (1972).

[10] B. Talin et. al., Phys. Rev. A 11, 648 (1975).

[11] V. Tsytovich, Comments Plasma Phys. Cont. Fusion, 4, 81 (1978).

[12] A. Sh. Abdullaev, Sov. J. Plasma Phys. 14214 (1988).

[13] V. Berezhiani and S. M. Mahajan, Phys. Rev. E 55995 (1997).

[14] M. N. Rosenbluth and C. S. Liu, Phys. Rev. Lett. 29, 701 (1972); J. M. Rax and N. J. Fisch, Phys. Fluids B 5, 2578 (1993).

[15] W. L. Kruer, The Physics of Laser Plasma Interactions (Addison-Wesley, Reading, MA, 1988).

[16] V. P. Silin, Sov. Phys. JETP 20, 1510 (1965); C. D. Decker et. al., Phys. Plasmas 1, 4043 (1994). 
[17] J. Dawson and C. Oberman, Phys. Fluids 5, 517 (1962).

[18] G. Shvets and N. J. Fisch, Phys. Plas. 4, 428 (1997).

[19] N. J. Fisch, Phys. Rev. Lett. 41, 873 (1978).

[20] N. J. Fisch and A. H. Boozer, Phys. Rev. Lett. 45, 720 (1980).

[21] N. J. Fisch, Rev. Mod. Phys. 59 (1), 175 (1987).

[22] Y. R. Shen, The Principles of Nonlinear Optics, John Wiley and Sons, New York, 1984.

[23] A. I. Alexeev, Opt. Spectrosc. 75, 499 (1993).

[24] W. B. Mori and T. Katsouleas, Phys. Rev. Lett. 69, 3495 (1992). 


\section{FIGURES}

FIG. $\quad 1 . \quad$ Azimuthal particle displacement in the prescribed laser field with $a_{0}(r)=0.1 \exp \left(-r^{2} / 2 \sigma^{2}\right)[1+\tanh \zeta / T]$. Solid line: no collisions $(\gamma=0)$; dashed line: $\gamma / \omega=0.1$; thin solid line: guiding center drift. Laser parameters: $T=10, \sigma=15 c / \omega$. Electron is launched at $\zeta=-4 T$ at $r=\sigma / 3$ with a vanishing velocity. 


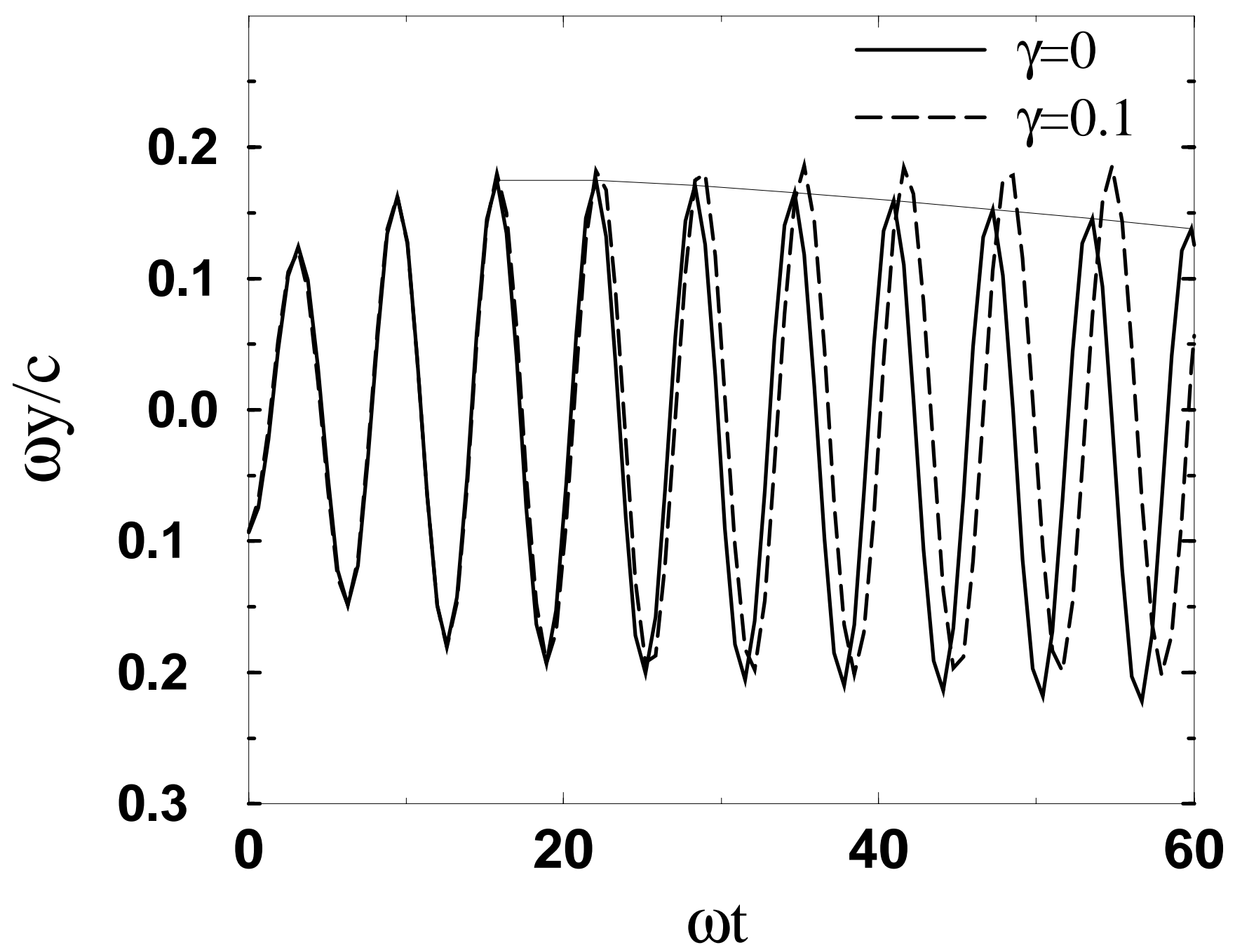




\section{External Distribution}

Plasma Research Laboratory, Australian National University, Australia

Professor I.R. J ones, Flinders University, Australia

Professor J oão Canalle, Instituto de Fisica DEQ/IF - UERJ , Brazil

Mr. Gerson O. Ludwig, Instituto Nacional de Pesquisas, Brazil

Dr. P.H. Sakanaka, Instituto Fisica, Brazil

The Librarian, Culham Laboratory, England

Library, R61, Rutherford Appleton Laboratory, England

Mrs. S.A. Hutchinson, JET Library, England

Professor M.N. Bussac, Ecole Polytechnique, France

Librarian, Max-Planck-Institut für Plasmaphysik, Germany

J olan Moldvai, Reports Library, MTA KFKI-ATKI, Hungary

Dr. P. Kaw, Institute for Plasma Research, India

Ms. P.J . Pathak, Librarian, Insitute for Plasma Research, India

Ms. Clelia De Palo, Associazione EURATOM-ENEA, I taly

Dr. G. Grosso, Instituto di Fisica del Plasma, Italy

Librarian, Naka Fusion Research Establishment, J AERI, J apan

Library, Plasma Physics Laboratory, Kyoto University, J apan

Research Information Center, National Institute for Fusion Science, J apan

Dr. O. Mitarai, Kyushu Tokai University, J apan

Library, Academia Sinica, Institute of Plasma Physics, People's Republic of China

Shih-Tung Tsai, Institute of Physics, Chinese Academy of Sciences, People's Republic of China

Dr. S. Mirnov, TRINITI, Troitsk, Russian Federation, Russia

Dr. V.S. Strelkov, Kurchatov Institute, Russian Federation, Russia

Professor Peter Lukac, Katedra Fyziky Plazmy MFF UK, Mlynska dolina F-2, Komenskeho Univerzita, SK-842 15 Bratislava, Slovakia

Dr. G.S. Lee, Korea Basic Science Institute, South Korea

Mr. Dennis Bruggink, Fusion Library, University of Wisconsin, USA

Institute for Plasma Research, University of Maryland, USA

Librarian, Fusion Energy Division, Oak Ridge National Laboratory, USA

Librarian, Institute of Fusion Studies, University of Texas, USA

Librarian, Magnetic Fusion Program, Lawrence Livermore National Laboratory, USA

Library, General Atomics, USA

Plasma Physics Group, Fusion Energy Research Program, University of California at San Diego, USA

Plasma Physics Library, Columbia University, USA

Alkesh Punjabi, Center for Fusion Research and Training, Hampton University, USA

Dr. W.M. Stacey, Fusion Research Center, Georgia Institute of Technology, USA

Dr. J ohn Willis, U.S. Department of Energy, Office of Fusion Energy Sciences, USA

Mr. Paul H. Wright, Indianapolis, Indiana, USA 
The Princeton Plasma Physics Laboratory is operated by Princeton University under contract with the U.S. Department of Energy.

\author{
Information Services \\ Princeton Plasma Physics Laboratory \\ P.O. Box 451 \\ Princeton, NJ 08543
}

Phone: 609-243-2750

Fax: 609-243-2751

e-mail: pppl_info@pppl.gov

Internet Address: http://www.pppl.gov 